\title{
CiUdad EduCADORA, DeSDe la Relación: EDUCACIÓN, INTEGRACIÓN, CIUDAD Y COMUNICACIÓN
}

Educative City, from the Relationship of Education, Integration, City and Communication

\author{
Marc Pallarès Piquer \\ pallarem@edu.uji.es \\ Universitat Jaume I \\ Josu Ahedo \\ josu.ahedo@unir.net \\ UNIR \\ Jordi Planella \\ jplanella@uoc.edu \\ UOC
}

RESUMEN: Si se acepta que una ciudad es algo más que una conjunción de espacios urbanos, se puede reivindicar la constitución de la ciudad educadora como proyecto pedagógico. El artículo reivindica un proyecto que percibe la ciudad como espacio-objeto de atención y como acción socio-pedagógica. La propuesta responde a un constructo social y educativo que articula la ciudad con una doble condición: como ámbito de conocimiento y como ámbito de acción. Se trata de la idea de acción como finalidad orientada a la consecución de expectativas y logros. Para tal finalidad, hay que entender la ciudad como un elemento cognoscible desde el que se crean conocimientos transculturales obtenidos de la realidad. Esto hace posible proponer experiencias que faciliten el contacto directo con la ciudad. Así, la propuesta se postula a favor de la ciudad como agente educativo, como eje de integración, como fuente permanente de información y como medio didáctico que proporciona aprendizajes. Se llega a la conclusión de que la ciudad educadora debe indagar en la vertiente educativa del espacio urbano y demandar una formación social basada en valores éticos que proporcionen al alumnado los valores de su acción y (una parte) del reconocimiento como persona social. 
Palabras Clave: ciudad educadora, educación, pedagogía, ciudadanía, escuela y comunidad.

ABSTRACT: If we accept that a city is more than simply an agglomeration of urban areas, the city of education as a pedagogical project is a valid demand. This paper calls for a project that perceives the city as a space-object of attention and as a socio-pedagogic action. This proposal responds to a social and educational construct with a dual conception of the city: as a sphere of knowledge and as a sphere of action. Action is understood as a purpose aimed at attaining expectations and achievements. To achieve this purpose, the city must be understood to be knowable, an element from which transcultural knowledge obtained from the reality can be created, thereby proposing experiences that encourage direct contact with the city. Thus, the proposal calls for the city to be an educational agent, a hub for integration, a continuous source of information and a didactic means for learning. The paper concludes that the city of education must inquire into the educational aspect of urban areas and demand a social training based on ethical values that provide students with values for their action and (a part of) their acknowledgment as a social person.

KEYWORDS: city of education, education, pedagogy, citizenship, school and community.

RESUM: Si s'accepta que una ciutat és més que una conjunció d'espais urbans, es pot reivindicar la constitució de la ciutat educadora com a projecte pedagògic. L'article reivindica un projecte que percep la ciutat com a espai-objecte d'atenció i com a acció socio-pedagògica. La proposta respon a un constructe social i educatiu que articula la ciutat amb una doble condició: com a àmbit de coneixement i com a àmbit d'acció. Es tracta de la idea d'acció com a finalitat orientada a la consecució d'expectatives i èxits. Per a tal finalitat, cal entendre la ciutat com un element cognoscible des del qual es creen coneixements transculturals obtinguts de la realitat. Això fa possible proposar experiències que faciliten el contacte directe amb la ciutat. Així, la proposta es postula a favor de la ciutat com a agent educatiu, com a eix d'integració, com a font permanent d'informació i com a mitjà didàctic que proporciona aprenentatges. S'arriba a la conclusió que la ciutat educadora ha d'indagar en la vessant 
educativa de l'espai urbà i demanar una formació social basada en valors ètics que proporcionen a l'alumnat els valors de la seva acció i (una part) del reconeixement com a persona social.

Paraules clau: ciutat educadora, educació, pedagogia, ciutadania, escola i comunitat.

La ciudad es la gente.

Sófocles

\section{Introducción}

Los tiempos en los que vivimos requieren debates educativos basados en la proposición de espacios que superen los clásicos límites de la institución escolar. Los paradigmas tradicionales de espacio y tiempo han sufrido transformaciones relevantes en las dos últimas décadas. En consecuencia, se pueden apreciar «cambios en la naturaleza del saber, su organización, su producción, su legitimidad, en los modos de comunicarlo y, específicamente, en su enseñabilidad» (Arellano, 2005, p. 80). Unos cambios que conllevan que la caracterización del profesional de la enseñanza contenga una referencia a la interdisciplinariedad, y también algunas indicaciones para interpretar correctamente su sentido (Ahedo, 2016).

Además, hay que tener presente que los centros escolares se encuentran ubicados en ciudades cada vez más fragmentadas, más «atomizadas y divididas en las cuales la precariedad, el individualismo, el conflicto y la pobreza son rasgos que las caracterizan» (Rodríguez y Díez, 2017, p. 79). Sin embargo, también contamos con unas ciudades más preparadas para disfrutar de ellas, es decir, no son sólo lugares para trabajar y dormir.

A partir de este contexto socioeconómico, no está de más recordar (y reivindicar) que «la escuela es un espacio privilegiado para desarrollar el concepto clave de comunidad democrática, convirtiéndose en un modelo organizativo transportable a la sociedad civil y, por tanto, en un agente activo 
de la reconstrucción social» (Lozano, Traver y Sales, 2016, p. 15). Esto que se propone aquí ya fue señalado por Dewey, la escuela democrática como lugar de aprendizaje de lo que es la democracia. Asimismo, la creación y el fomento de estrategias participativas resultarán imprescindibles para la eficacia de la gestión urbana y para la democratización de las estructuras de gobierno.

A raíz de lo descrito, este artículo va a ocuparse de justificar que la ciudad no es únicamente un conjunto urbanístico; la ciudad se encuentra conformada por estructuras físicas y por elementos que establecen sinergias producidas entre las instituciones y las dimensiones culturales, hecho que ofrece a la acción educativa la oportunidad de aprender en la ciudad y de la ciudad; para ello se necesitan unos condicionantes: la consolidación de una sociedad civil sólida, conformada por personas participativas y activas, por ejemplo, pero también se requiere de aspectos como un cambio de visión y de estrategia por parte de ciertas administraciones públicas.

Planteamos, por consiguiente, la necesidad de desarrollar la concepción de educación urbana entendida como «las actividades educativas desarrolladas en el contexto de la administración local» (Colom, citado en Rodríguez, 2000, p. 129).

De esta manera, se abre la posibilidad de que la ciudad se convierta en ciudad educadora, entendida como sugerencia pedagógica ${ }^{1}$ pero también, en

1. Aunque el concepto de ciudad educadora «reposa sobre tres pilares: $a$ ) buena comunicación de las oportunidades que ofrece la ciudad a todos y cada uno de los ciudadanos. En lo referente a los gobiernos locales, quiere decir, además la explicación del porqué y del cómo de sus políticas, es decir, de hacer de la política, pedagogía; b) participación corresponsable de los ciudadanos: definiendo y acordando previamente, el alcance, los límites y los campos; c) evaluación del impacto educativo de las diferentes políticas y también del grado de utilidad y de eficacia de éstas» (Figueras, 2008, p. 20), el presente artículo se centra en el primero de ellos para, así, postularnos a favor de una ciudad entendida como pedagogía. 
cierta manera, como propuesta política, ${ }^{2}$ puesto que el/la «ser ciudadano/a», según la concepción aristotélica, es que aquella persona puede participar de las diferentes funciones deliberativas de la ciudad. No es posible una sin la otra. Se aprende de la ciudad y uno de los aspectos que hay que aprender es a ser ciudadano/a (aunque éste no es el punto esencial de nuestro artículo). Como afirman Borja y Castells (1997), el ciudadano o la ciudadana es quien se ha involucrado en la conquista ${ }^{3}$ y la configuración de la ciudad, por eso «ser ciudadano no es una condición que se alcanza al llegar a una determinada edad; es la práctica continua de ciertos valores que el ser humano debe encontrar en la ciudad en la que habita» (Rodríguez, 2007, p. 30).

Subyace, en esta última cita, la idea Heideggeriana según la cual «el simple hecho de vivir no es todavía habitar, pues el hombre, cuando habita, habita de acuerdo con la frase de Hölderlin: poéticamente sobre esta tierra» (Heidegger, 1989, p. 69). Por tanto, ser ciudadano/a es más que ser residente, conlleva un compromiso con la ciudad.

A este compromiso vamos a dedicar las presentes páginas, pues, en pleno siglo XXI, uno de los requisitos que se le puede exigir a la controvertida etiqueta de «ciudadanía» pasa por aspectos como el de la posibilidad de fijar prácticas pedagógicas establecidas en función de la conexión entre el ser humano y la ciudad; en cierta manera, se trata de prácticas pedagógicas que pueden hacer posible algo tan significativo como que «mientras ésta [la ciudad] lo ciudadaniza, aquél [el ser humano] la humaniza» (Rodríguez, 2007, p. 30). Y si este ejercicio que se propone es posible es porque el valor de la educación es un patrimonio cultural, que puede ser interpretado desde dife-

2. Estamos de acuerdo con Bryon y Gaona (2005, p. 134) cuando aseveran que «la educación y la pedagogía [...] deben ser entendidas como un acto político que configura la construcción del sujeto social. [...] En pocas palabras, [el maestro] es el encargado de dinamizar el mismo acto pedagógico, el cual es siempre un acto político», entendiendo como político simplemente aquello que habilita a la pedagogía como «una concepción de la verdad que resulta firme porque no se entienda a partir de las certezas de una serie de análisis hechos a medida (para una realidad contextual muy concreta), sino a partir de las imperfecciones del episteme, esto es, de aquellas verdades que lo son porque encuentran más resistencia para ser aceptadas que cualquier otra pauta histórica de verificación estratégicamente establecida» (Pallarès y Traver, 2017).

3. El propio Borja tiene una obra titulada Ciudad conquistada (2003), donde define la ciudad como un espacio público en el que tienen que confluir elementos de redistribución social, cohesión comunitaria y autoestima societaria. 
rentes dimensiones, en el que las sociedades aceptan, transforman y difunden las distintas formas simbólicas y culturales de las que disponen, así como sus prácticas pedagógicas y sus maneras de vida.

A la postre, una ciudad educadora pedagógicamente factible será aquélla que aceptará y aunará la educación formal, la no-formal y la informal, y que se erigirá en un eje de actuación derivado de «la dialéctica entre lo pedagógicamente ordenado y el inevitable juego de encuentros y vivencias educativas que se producen por el hiper-complejo medio urbano» (Trilla, 1997, p. 22), una intersección entre encuentros y vivencias acaecida en un espacio urbano que dispone de suficientes alicientes para crear escenarios que vehiculen elementos y acciones con posibilidades educativas.

La idea de ciudad educadora fue propuesta ya por Edgar Faure en el año 1972 en la obra colectiva Apprendre à être. Partimos de esa idea, puesto que proponemos un interés por las cualidades sociales - y no sólo físicas- de los lugares, esto es, por la búsqueda de espacios urbanos como escenarios de acción educativa. Pero también nos referimos a la promoción de actividades culturales y a la expansión de decisiones destinadas a un enfoque de la escena pedagógico-social en la que acción escolar, identidad, patrimonio cultural y $\operatorname{conflictos}^{4}$ (vividos democráticamente, por supuesto) sugieren y fijan otro(s) modo(s) de vida, una posibilidad alternativa de vivir (mejor) como ciudadanía, en definitiva.

\section{La ciudad no es sólo una urbe: más allá de la suma de las peri- ferias y los barrios ${ }^{5}$ céntricos}

La premisa fundamental para postularnos a favor de lo apuntado en la introducción es percibir que una ciudad es algo más que una conjunción de

4. En este sentido, para no caer en propuestas quiméricas, es interesante prestar atención a las palabras de Rodríguez (2000, p. 127) cuando asevera que asistimos a transformaciones en la mirada sobre la ciudad que nos llevan a un «lugar en donde se encuentra la solución para problemas de la vida, hasta convertirse en un lugar-problema por excelencia»».

5. La palabra barrio dispone de diferentes acepciones y, además, su significado se ha ido modificando a lo largo del tiempo (Beltrán, 2017). Blackson (2012) explica que un barrio es una herramienta óptima para definir y estructurar las políticas y ordenanzas necesarias para conservar, mejorar y planificar entornos socialmente activos y sanos. 
espacios urbanos conformada por una serie de mobiliario urbano, por sus periferias (caracterizadas por ser espacios alejados, tanto desde un punto de vista espacial como relacional), por los barrios más céntricos, etc.

Calonge (2017, p. 152) asegura que:

En el contexto neoliberal presente, la mano invisible del mercado inmobiliario es el mecanismo que induce que esta ocupación de la periferia se realice de manera segregada. Los habitantes de menores ingresos no encuentran otro espacio que el periférico para residir, ante la subida generalizada de los valores inmobiliarios en la ciudad consolidada.

He aquí, seguramente, uno de los retos más importantes de la ciudad educadora, aunque escape de los objetivos de este artículo: aportar argumentos y facilitar el desarrollo de actuaciones encargadas de minimizar la instauración de la dualidad «ciudad consolidada-ciudad abandonada» que encontramos en muchas de las ciudades actuales. El fenómeno del contraste «barrios periféricos-barrios céntricos» ha sido una temática que ha contado con una atención especial por parte de la sociología en las últimas décadas. Dichos estudios se han centrado en la disposición del hábitat, pero también en las relaciones sociales de estos entornos, y en la idiosincrasia y la caracterización de las conexiones espaciales que se producen entre las periferias y el resto de la ciudad (Jacquin, 2012).

A la premisa a la que aludíamos al inicio de este apartado hay que añadir que entendemos y aceptamos que lo educativo de la escuela no comienza y acaba en ella, lo educativo empieza «en la complejidad de las acciones humanas, en el mundo de la vida y en la producción de sus vidas totales, por eso [en la acción educativa] hay que ir más allá de la escuela» (Moll, 2000, p. 10): podemos aprender de la ciudad, desde sus museos, con su patrimonio, etc., y se puede llevar a cabo a través de actividades pedagógicas que despierten posibilidades de acción educativa (Walker y Chaplin, 2002).

El punto de partida es, por lo tanto, la conjunción de estas dos premisas, que nos permitirán proponer una dimensión pedagógico-social que amplíe el sentido de los procesos educativos (demasiado encerrados en los muros escolares y en las posibilidades que ofrecen otras vías, como internet, que si bien es cierto que, de alguna manera, abren la escuela al mundo no lo es 
menos que no deberían impedir que la acción educativa conectara con su entorno más próximo a través de salidas por la ciudad con nuestro alumnado, etc.). Estamos hablando, tal y como ya lo hizo de modo explícito la Carta de Ciudades Educadoras de 2008:

Entre otros aspectos, de la necesidad de la construcción y reconstrucción de los lazos o vínculos entre las personas; el fomento de una convivencia hospitalaria y amistosa; la cooperación mutuamente enriquecedora para facilitar la autoestima individual y colectiva; el desarrollo de las potencialidades latentes y el uso adecuado de las aptitudes de cada uno/a; todo lo cual queda resumido en la idea, literalmente expresada en el sentido de «reconstrucción del espacio público» como diálogo constante entre individuo y comunidad y entre derechos y deberes de todos los actores cívicos; y una educación a lo largo de la vida que no solo renueve habilidades para el cambio laboral y técnico, sino sobre todo para la renovación del espíritu de ciudadanía. (Del Pozo, 2008, p. 28)

En relación a lo que apunta esta cita conviene señalar que la reivindicación de la ciudad educadora debe comportar necesidad de «transformar» a las personas de objetos pasivos y dependientes en sujetos activos (Pérez, 2005). También hay que recordar que la dimensión urbanística lleva casi dos siglos proclamando (desde una autonomía casi «total») que el constructo urbano debía ser quien estructurara (y quien condicionara) las formas de vida; incluso ha ido forjando aspectos tan esenciales como el de la identidad; esto ha propiciado que la ciudadanía, sobre todo a lo largo del desarrollo urbano de la segunda mitad del siglo $\mathrm{xx}$, «se apropie de la historia urbana y participe de ella con el fin de hacer de la urbe un lugar vivible» ${ }^{6}$ (Pérez, 2005, p. 131).

De hecho, Robert Park (2000, p. 3) afirmaba que en realidad la ciudad es el lugar donde:

La humanidad ha intentado recrear el mundo de una forma más consecuente con sus deseos más profundos y donde, en general, mejor lo ha conseguido. Pero si la ciudad es el mundo que ha creado el hombre, también es el mundo en el que está condenado a vivir. De modo que, indirectamente y sin una idea clara de la naturaleza del intento, al crear la ciudad, el hombre se ha recreado a sí mismo.

6. Las cursivas son nuestras. 
Al afirmar que «el hombre se ha recreado a sí mismo», en estas palabras de Park, subyace una especie de utopía dialéctica (Pallarès y Planella, 2016), ya que, en cierta manera, no hay orden social alguno que se pueda transformar si no contiene, aunque sea de manera indirecta, algunas de las dimensiones de lo que se quiere instaurar (de lo «nuevo»). En este sentido, Harvey (2008, p. 48 ) asegura que «las revoluciones no son una ruptura completa, sino que lo que hacen es darle la vuelta a las cosas»».

El fomento y la implementación de prácticas educativas insertadas en la ciudad educadora viene a ser como un segundo paso: tras la preocupación por hacer de las ciudades unos lugares vivibles a la que hacía referencia Pérez en la cita anterior, se trata ahora de requerir la inclusión de la combinación del sistema educativo formal en un acervo de relaciones (y decisiones) políticas y sociales nuevas (Paupeiro, 2004).

Esta combinación hay que llevarla a cabo con el objetivo de que la ciudad no sólo sea un medio sino también un objeto fundamental de aprendizaje. Hay que aprender con la ciudad, pero también de la ciudad. Por consiguiente, proponemos concebir la ciudad como una intersección donde confluyan los saberes mediante diseños curriculares y prácticas educativas capaces de aunar una idea de lo humano con base en sus constructos culturales nucleares: moral (ética), expresiva y cognoscitivo-instrumental (ciencia y técnica) (Giraldo, 1995); en consecuencia, hay que ubicar a la escuela, al alumnado y al resto de la ciudadanía en un contexto capaz de generar alternativas sociales y culturales, esto es, «se trata de desarrollar la personalidad histórica de la escuela y de la cultura y, para ello, la pedagogía desempeña un papel de primer orden» (Rodríguez, 2008, p. 7).

De esta manera, se busca que la ciudad ya no sea una amalgama urbanística y de personas que cohabitan sino «una gran alma, una ciudad viva, un cuerpo que siente, que se mueve, una ciudad con corazón propio, un ambiente y un contexto global de vida y aprendizaje» (Rodríguez, 2006, p. 47).

Un ejemplo de ello puede ser la ciudad italiana de Turín, donde en el año 1975:

[...] desarrollamos una idea revolucionaria: la ciudad podía ofrecer a la escuela espacios y oportunidades normales, y no en miniatura, con el fin de enriquecer las potencialidades formativas de las escuelas y al mismo tiempo hacer 
comprender, amar y defender, desde la perspectiva de los adolescentes, su nueva ciudad. ${ }^{7}$ El alcalde de entonces, Diego Novelli, y los concejales nos dirigimos antes que a las escuelas y a los profesores, a los distintos grupos extra-escolásticos de la ciudad con estas palabras: «El correcto funcionamiento de nuestro sistema educativo representa un interés común; la escuela no puede sola; cada uno de ustedes debe prepararse para dar su aporte contribuyendo con situaciones y personas que puedan revelarse como útiles e interesantes». [...] Los teatros tenían interés por preparar públicos nuevos; las bibliotecas porque los ciudadanos se apasionaran por la lectura desde niños; las industrias porque el ciclo productivo se conociera y se valorara; las asociaciones deportivas porque la gente se acostumbrara a amar el ejercicio físico dirigido; las organizaciones naturalistas porque se desarrollara el conocimiento y el respeto por las plantas y animales; los bomberos porque los ciudadanos hicieran una prevención adecuada; la asociación de panaderos porque se valorara el buen pan; y hasta [...] la policía porque para que [el alumnado] fueran conscientes y respetuosos con su trabajo. [...] De la gran cantidad de trabajo realizado en los primeros años surgió una idea aún más ambiciosa: transformar la ciudad — puesta a disposición de la escuela—en una verdadera ciudad educadora. (Alfieri, 2005, p. 33)

Aunque no se va a entrar en el debate sobre las diferentes posibilidades que pueden ofrecernos (social y educativamente) las transformaciones de las ciudades, en este sentido, Harvey (2008) asegura que el derecho a transformar la ciudad no es abstracto, sino que es un derecho que se lleva a cabo en nuestra cotidianidad; la dialéctica de la urbanización y la de la transformación social se desarrolla continuamente a nuestro alrededor, por eso nos relacionamos con los otros, construimos o nos movemos por los distintos entornos urbanos «y colaboramos, bien, mal o regular, en sus consecuencias» (Harvey, 2008, p. 45).

7. Este proyecto se llamó La città ai bambini. A finales de los 70 lo que sucedió es que:

Puse a disposición de los amigos catalanes $-\mathrm{y}$ más tarde de Madrid, Valencia y Santiago de Compostela» las experiencias pedagógico-didácticas [...] de mi ciudad. Cuando en el sistema escolar-educativo italiano las ideas innovadoras al interior y al exterior de la escuela se difundían con relativa dificultad, en Cataluña y por toda España la difusión fue extraordinariamente rápida. Por una parte empezó el proceso que dio lugar a la gran reforma escolar en España [...], y de otra parte se desarrolló la idea de la ciudad educadora. Y precisamente en Barcelona, en noviembre de 1990, tuvo lugar el primer congreso mundial sobre este tema que concluyó con la adopción de la Carta de las Ciudades Educadoras (Alfieri, 2005, p. 37). 
Nos limitamos, tal y como hemos visto con el ejemplo de la ciudad de Turín, a reivindicar la capacidad que tiene la ciudad para devenir un elemento al servicio del aprendizaje significativo; y lo hacemos con base en aceptar la educación como un compendio entre conocimiento y formación. Hablamos, por lo tanto, de implementar prácticas que consigan que nuestro alumnado interprete una parte de la realidad a partir de su entorno más próximo. Proponemos, en definitiva, que concebir, ordenar y relacionar a la ciudad con algunos de los saberes y contenidos escolares satisface las exigencias praxiológicas de la actividad educativa: la ciudad se convierte en un agente educativo, porque contiene información teórico-práctica fiable, y el alumnado aprende de ella. Pero nuestra propuesta va más allá. Hay que aprender de la ciudad, aprender desligado de la urbe en la que se vive es desnortar lo aprendido y desnaturalizarlo.

Es una propuesta que consideramos que se encuentra en continuo crecimiento, un espacio pedagógico que hay que ir recorriendo para poder materializar las ideas y propuestas de los estamentos que constituyen el tejido social de la ciudad. Se trata, pues, de un proyecto para crear ciudadanía y democracia: construir ciudad para más y mejores ciudadanos/as, y su eje vertebrador es la configuración de una nueva ciudadanía (Rodríguez, 2007). Siempre teniendo presente que ser ciudadano/a no implica sólo ejercer los derechos civiles sino comprometerse a mejorar la ciudad, responsabilizarse con ella, etc. Todo ello sin olvidar el papel de las administraciones locales, que «pasan a asumir la categoría de instituciones políticas con capacidad para establecer espacios de educación para la democracia» (Cabezudo, 2005, p. $85)$.

Aunque no pretendemos convertirla en una vía de resolución de problemáticas sociales de diferente índole, una propuesta como ésta que desarrollamos «puede aspirar a generar un clima de calidad cívica y de convivencia urbana» (Del Pozo, 2008, p. 28), esto es, a concebir el medio urbano como entorno, como agente y como contenido de la educación.

En esencia, la propuesta de ciudad educadora implica entender la escuela como algo más que un agente de socialización. Como el objetivo de la escuela no puede reducirse a la socialización, se contempla la ciudad como un ámbito de aprendizaje porque el alumnado reside en una ciudad y no tiene sentido 
desligar lo aprendido en la escuela de lo que se aprende con la convivencia en la ciudad. Por lo tanto, hay que potenciar la capacidad del alumnado para ser sujeto, ${ }^{8}$ esto es, apostar por unas prácticas pedagógicas y proporcionar unas herramientas de formación que hagan posible que el alumnado disponga de un importante grado de autonomía. Dicha autonomía se desarrolla a partir del contacto directo con el espacio urbano, y requiere «consolidar la organización y la presencia de los agentes o grupos sociales que forman la red social de la ciudad» (Pérez, 2005, p. 136). Es una autonomía que, a partir de su desarrollo como eje desde el que el alumnado integra lo urbano en la escuela, hará posible el desarrollo de la formación humana y colaborará en la tarea de profundización de los valores y en la axiología cívica.

La dimensión del aspecto social de la ciudad al que aludía Pérez en la cita anterior implica la interacción e integración social, ${ }^{9}$ y engloba dimensiones que potencian la «vitalidad» como son la convivencia de las diferentes clases sociales, una comunidad cercana o la adaptación a los denominados alejamientos sociales (Beltrán, 2017, p. 31).

Apostar por un proyecto que incluya a la ciudad educadora, por lo tanto, es ampliar el campo de la pedagogía teniendo en cuenta a la ciudad como espacio-objeto de atención y como acción socio-pedagógica. De esta manera, a través de programas de educación compensatoria, sanitarios, preventivos, asistenciales y también mediante la enseñanza de los contenidos culturales que aporta la ciudad, así como a través de estudios sobre formas urbanas y de vida de las ciudades, se pueden llevar a cabo acciones orientadas a la optimización pedagógica del ejercicio docente y de otras instituciones consideradas por la «educación permanente» como educadoras de las personas a lo largo de la vida.

8. Con esto reforzamos nuestra idea de una ciudad educadora basada en una acción educativa que se decanta por construir la democracia y la ciudadanía como un principio vital del ser humano.

9. Es por ello que el apartado tercero del presente artículo se ocupará de la integración. 


\section{La ciudad como desafío de integración de las personas}

En lo que se refiere a la integración, la ciudad puede llevar el desarrollo de dos tendencias: «una estrategia de planificación de la vida urbana y su construcción voluntaria, o un proceso de guetización y fragmentación, que desemboca en un proceso de marginalidad» (Bryon y Gaona, 2005, p. 130). La primera genera procesos que contribuyen con la cohesión del tejido social; la segunda establece condiciones que van creando ciudades cuyos barrios cada vez son menos heterogéneos.

Para defender, fijar y promover la primera de estas tendencias se hace necesario entender que la educación es un derecho de las personas, hecho que implica una singularidad previa a cualquier estructuración de política educativa, a cualquier propuesta de reforma educativa y a toda práctica diaria de aula (Gimeno, 2013).

A partir de la aceptación de este derecho, consideramos que la ciudad, convertida en un reto para la integración, es un escenario de conquista social que atañe a toda la ciudadanía (en caso contrario, se pueden producir actuaciones que desemboquen en marginaciones, alejamientos, disfunciones sociales, etc.). Este escenario de logro social conlleva entender la pedagogía como una disciplina habilitada para configurar proposiciones teóricas sustantivas de la educación y prácticas de intervención enmarcadas, en cada caso particular, en la racionalidad que mejor se adapte a la complejidad del objeto de conocimiento de cada acción educativa concreta (Touriñán y Sáez, 2015). Así, la ciudad educadora interacciona, se nutre, aporta y se ensancha en otros sistemas: patrimonio cultural, medio rural, interacción con otras ciudades, etc.

Otro de los aspectos clave es el de los alejamientos, que en ocasiones son meramente físicos (respecto a los epicentros urbanos, que, con la acumulación de capitales y de recursos, terminan implicando otra serie de alejamientos respecto a los escenarios para la inclusión social). Como muy bien han demostrado Dickerson (2008) y Lindón (1997), la segregación espacial actúa como un mecanismo para la canalización diferencial de recursos por los diferentes grupos y clases sociales. Estar alojado en una periferia, en la mayoría de ciudades, puede implicar quedar excluido de los círculos económicos y sociales más básicos de la ciudad (Calonge, 2017). 
El fomento de la relación escuela-ciudad (y las diferentes formas de acción que conllevará el impulso de la ciudad educadora) exigen una interrelación entre la pedagogía y las políticas públicas. La ciudad es una conglomeración de espacios públicos en el que se tiene que manifestar la democratización social y, por consiguiente, el estatus de ciudadanía implica entender la ciudadanía como un conjunto de sujetos políticos activos, con el derecho y la opción de acceder a la diversidad de las ofertas urbanas (Bryon y Gaona, 2005).

Una de las premisas esenciales para poder hablar de ciudad educadora es que la Pedagogía ponga al servicio de la acción educativa el acceso a esta diversidad de ofertas urbanas, de manera que el concepto de ciudad educadora se refiera a «un medio que produce relaciones y efectos educativos premeditados» (Limón y Crespo, 2002, p. 93). Algunos estudios urbanos recientes (Pinder, 2011; Strohmayer, 2011; Thrift, 2008) han evidenciado la urgencia por recuperar una perspectiva dinámica sobre el espacio «que restituya su sentido procesual y energético» (Calonge, 2017, p. 168). Según estas nuevas propuestas, la mayoría de los acontecimientos relacionados con la movilidad son cruciales para «superar la visión fija y estática de los marcos de referencia temporales» (Malpas, 2004, p. 165). En esta recuperación, las prácticas socio-educativas en general y las prácticas de movilidad en particular son uno de los elementos nucleares, porque «son ellas mismas generadoras de espacio y tiempo» (Calonge, 2017, p. 168).

Sin embargo, hablamos de la generación de espacio y tiempo ${ }^{10}$ como unos ejes que faciliten una serie de conocimientos desde los que poder adoptar decisiones (pedagógicas) que favorezcan la integración, desde los que seamos capaces de plantear un conjunto sistemático de recomendaciones que, en definitiva, informen a quien asume funciones pedagógicas sobre cómo alcanzar los objetivos marcados.

El hecho de hablar de integración, ya sea en el ámbito educativo o en cualquier otro, supone ubicar a las personas, habitantes de la ciudad, en el centro,

10. Estamos de acuerdo con Romero (2004, p. 123) cuando explicita que para que la educación se asuma como un proyecto temporal «el proyecto educador debe semantizar de otro modo el tiempo: el futuro como exigencia de determinabilidad de lo indeterminado y no probabilístico». 
tanto en su dimensión afectiva como en una esfera de propuestas que exijan como punto de inflexión la creación de nuevos procesos de subjetivación (que reforzarán la condición sistémica de lo educativo y exigirán al profesional docente dinámicas de trabajo y acciones pedagógicas integradoras). En este sentido, pero de manera más concreta:

Se resalta la importancia de fortalecer y de promover alternativas de formación y participación para el maestro, desde su saber y reconocimiento. Si bien la Ciudad Educadora convoca a muchos actores y agentes educativos, no en vano el papel y rol del maestro y de la escuela se constituyen en vectores de fuerza y no simplemente en nodos de una trama que juega en varios planos de acción.

(Castro, 2005, p. 73)

De la misma manera que en muchas ocasiones ha sido la sociedad la que «ha generado parámetros de segregación y exclusión social ${ }^{11}$ que después han sido reproducidos por las instituciones educativas» (Torres, 2009, p. 31), desde estas páginas proponemos planes de desarrollo educativo que, mediante situaciones, espacios y elementos vivenciales de la ciudad educadora, promuevan visiones más completas de la diversidad. La integración no es sólo una cuestión educativa, en el sentido pedagógico, aprender a respetar al otro, sino que precisa que la dimensión espacial y temporal de la ciudad también contribuyan a esta integración. Si los espacios urbanísticos no son integradores, entonces lo que sucede es que la pedagogía no se mueve en el mismo sentido que la dimensión urbanística.

Los acontecimientos simplemente suceden, pero las propuestas que ambicionan mejorar algún aspecto socio-educativo terminan siendo eficaces en

11. Torres (2009, p. 35) pone de manifiesto que son las propias sociedades las que han generado prácticas educativas que «han ido estableciendo parámetros que fomentaban la exclusión de tal manera que ésta se ha convertido en un grave problema en nuestro mundo actual, que aunque se manifiesta en el ámbito educativo, familiar e institucional, es fundamentalmente un problema de índole social». De hecho, así lo evidencia Walker (1997, p. 8) cuando define la exclusión social:

Es un proceso dinámico que lleva a ser expulsado, total o parcialmente, de cualquiera de los sistemas social, económico, político y cultural que determinan la integración de una persona en la sociedad. La exclusión social también puede ser vista como una negación (no una realización) de los derechos civiles, políticos y sociales de los ciudadanos. 
función de su adecuación a las circunstancias que las «teorías» consiguen articular como significativas. Así, llevar a cabo con nuestro alumnado algunas experiencias metodológicas como el Aprendizaje-Servicio, por ejemplo, introducen a nuestro alumnado en la ciudad y ayudan a pluralizar fuentes y requisitos educativos nucleares. Con propuestas como esta lo que hacemos es facilitar la tarea de reestructuración de las relaciones espaciales urbanas en tanto que, el Aprendizaje-Servicio, se concibe como un método pedagógico basado en la experiencia (en el que el proceso de enseñanza-aprendizaje radica en la aplicación de lo que se va aprendiendo ante necesidades sociales reales). Fundamentado en la teoría del aprendizaje experiencial de Dewey (1938), el ApS se concibe como un método pedagógico que respeta una conexión operativa entre la teoría y la práctica, facilitando al alumnado la posibilidad de aprender a la vez que presta un servicio social. De este modo, el ApS promueve aprendizajes experienciales que, además, se ven reforzados por un importante componente de reflexión y crítica sobre las experiencias vividas (Domangue y Carson, 2008). Desde esta concepción, el ApS se apoya indiscutiblemente en la idea de Kolb (1984) de que el aprendizaje debe producirse en escenarios que coloquen al alumnado en situaciones y contextos conectados con su entorno cultural y social. De manera que, inevitablemente, su aplicación implica una praxis educativa instalada en situaciones y acontecimientos reales, procurando dar significatividad y genuinidad a los aprendizajes alcanzados

Específicamente, propuestas como ésta del Aprendizaje-Servicio son métodos pedagógicos experienciales definidos y en los que los estudiantes trabajan contenidos y objetivos curriculares mientras salen de los centros escolares y prestan un servicio significativo a la comunidad.

Hablamos, por lo tanto, de una propuesta que implica un tipo de actividades que unen aprendizaje con practicidad operativa en beneficio de la ciudad. Son prácticas educativas que, por un lado, ayudan a identificar las dificultades en la tarea de incorporar la inclusión en las instituciones escolares, $\mathrm{y}$, por el otro, establecen nuevos confines, ya que, a partir de la incursión de la acción 
pedagógica en la ciudad, la acción educativa queda habilitada para fijar de manera distinta las tradicionales nociones de fronteras e integración. ${ }^{12}$

En la concepción de ciudad educadora aportamos la idea de minimizar las desigualdades de manera parecida a los modelos clásicos, basados en dinámicas generales a partir de las cuales se cimentaba un orden social que gravitaba en el mérito, para poder, así, detectar las desigualdades y procurar subsanarlas. La apuesta de la ciudad educadora como vía para mejorar la integración se canaliza a partir de la perspectiva defendida por Touraine (1988, p. 278), según la cual se «introduce una visión realista y no idealizada de las situaciones colectivas y personales, y conduce, así, a reubicar los conocimientos - y lo que se denomina como "los valores" - en situaciones sociales e históricas concretas, al vincular ciencia y sociedad o ética». Esta concepción de la educación, en consecuencia, no se caracteriza únicamente por pertenecer a una sociedad democrática, sino que otorga a la escuela un rol activo de democratización al tener presente las condiciones concretas en que los niños y las niñas se ven expuestos a los mismos mecanismos y a los mismos problemas (Touraine, 1988).

Extrapolamos, aquí, todo lo aplicable al aula regular hacia la ciudad, para aceptar que la integración necesita «situarse en el corazón mismo de todo el trabajo de la escuela, siendo elemento esencial de la planificación del desarrollo, y llevado a cabo por todos los que tienen responsabilidad en el liderazgo y en la gestión escolar» (Ainscow, 2001, p. 2). Ponemos, en definitiva, la ciudad educadora al servicio de la integración, para evitar aquella escuela que a menudo «excluye unos tipos de conocimiento, propios de algunos grupos sociales, pugnando por una acomodación indolora a un tipo de conocimiento que le permitirá perpetuarse» (Bryon y Gaona, 2005, p. 135).

De esta manera, en lugar de indagar las vías para que la escuela se amolde a lo que la sociedad le exige lo que hacemos es proponer la ciudad como agente educativo, como fuente permanente de información y como medio didáctico que proporciona aprendizajes. Defendemos una ciudad educadora

12. El hecho de proponer acciones educativas concretas, como ésta del Aprendizaje-Servicio, permite que no caigamos en propuestas que «atienden más a la buena fe y la rutina que a la búsqueda afanosa de pautas de análisis intersubjetivas» (Touriñán y Sáez, 2015, p. 241). 
entendida como la base de un concepto de integración que no sólo determina la presencia física de (todo) el alumnado sino que propone fundamentos que permiten satisfacer las necesidades de los alumnos y alumnas, evitando etiquetas y otorgando los recursos y apoyos requeridos que «dentro del proceso de enseñanza y aprendizaje pueden presentar dificultades que obstaculicen su aprendizaje, lo que nos lleva a respetar sus diferencias, promover el desarrollo de habilidades cognoscitivas y de comunicación junto a sus pares en el aula regular» (Garnique y Gutiérrez, 2012, p. 580).

\section{La ciudad educadora como fuente de conocimiento y de ac- ción}

Después del recorrido que hemos llevado a cabo en el artículo, conviene tener presente que justificar y promover una ciudad educadora implica tener que preguntarse sobre la educación como objeto de conocimiento y como escenario de comunicación interhumano. Este hecho nos conduce a aceptar que «cuando hablamos de la educación como objeto de conocimiento [...] hay que preguntarse por qué determinados conocimientos se constituyen en meta e instrumento de la acción educativa, ${ }^{13}$ o por qué es educable la dimensión cognitiva del hombre» (Touriñán y Sáez, 2015, p. 242).

En función de esto, tal y como hemos comprobado con el ejemplo de Turín, determinamos que una propuesta como la de la ciudad educadora es un marco a partir del cual propagar los valores que son consecuencia de la dimensión y el sentido inherente al significado del concepto educación, pues se utiliza la ciudad como un contexto puesto al servicio de la finalidad de generar conocimientos, destrezas, actitudes y competencias en nuestro alumnado.

No obstante, la manera en que el alumnado vive sus procesos educativos en una ciudad adquiere particularidades diferentes, porque son constituidos a

13. En este sentido, hay que resaltar que «educar» no es sólo un proceso cognitivo de asimilación de contenidos, porque, si fuera esto exclusivamente, no resultaría sencillo encontrar su conexión con la ciudad educadora. 
lo largo de su existencia, por eso muchos de ellos son el resultado de una vida comunitaria rica en experiencia y sufrimientos (Pérez, 2005).

Como explica Morin (1998), una ciudad es una red compuesta de elementos heterogéneos asociados, capaz de presentar lo singular y lo múltiple. La complejidad de la ciudad viene constituida, para Morin, por aspectos tales como:

El tejido de eventos, acciones, interacciones, retroacciones, determinaciones, azares, que constituyen nuestro mundo fenoménico. Así es que la complejidad se muestra con los rasgos inquietantes de lo enredado, de lo inextricable, del desorden, la ambigüedad, la incertidumbre, el pensamiento complejo no es aquél que evita o suprime el desafío, sino aquél que ayuda a revelarlo e incluso, tal vez, a superarlo. (Rodríguez, 2007, p. 31)

En este sentido, no son pocos los autores que sitúan a la incertidumbre como elemento nuclear de la pedagogía, ${ }^{14}$ ya que no entienden la pedagogía como un intento (más) por estructurar un agente totalizador de saber que sirva para canalizar la formación y la enseñanza; por el contrario, la interpretan como «un despliegue de saberes que, en los fragmentos y los límites, conceptualiza los diferentes sentidos que aparecen. Desde allí, cobran vida los saberes, su producción y circulación, su enseñanza y su reflexión, condición para repensar los estilos y modos de hacer la Pedagogía» (Arellano, 2005, p. 83).

Para desarrollar una ciudad educadora en la que confluya el inter-juego entre proyectos personales, objetivos escolares y propósitos sociales se hace necesaria una comunicación real y efectiva entre los diferentes agentes sociales implicados. Hablamos de una comunicación entendida como el elemento de intercambio de reflexiones pedagógicas entre escuela, padres y madres, agentes sociales, empresas e instituciones públicas capaz de articular un eje en el que:

14. Se introduce aquí la «incertidumbre» porque Pallarès y Chiva $(2017$, p. 91$)$ hablan de que la «consolidación de la pedagogía se puede conseguir a partir de la reafirmación de la racionalidad de su discurso», una racionalidad que Gozálvez (2010, p. 39) asevera que «bien en su dimensión empírica, bien en su dimensión reflexiva, [...] ha de ser dialéctica más que analítica, es decir, abierta a la incertidumbre, la complejidad y los desórdenes de la realidad educativa». 
La educación se convierta en objeto de conocimiento, bien como pedagogía cognitiva o bien como didáctica, pero además de responder a por qué se produjo un determinado acontecimiento educativo y a cómo se puede lograr un determinado acontecimiento educativo, hay que responder a cómo se justifica ese acontecimiento educativo, y ésta es una cuestión que sólo se responde desde el conocimiento que tenemos del fenómeno educativo, en tanto que se construye desde la Pedagogía como ámbito de educación. Ésa es la pregunta desde la Pedagogía, no por mejorar nuestro modo de conocer, ni por mejorar nuestro modo de enseñar, sino la pregunta por la educación misma, [...] por la educación como tal, que es la pregunta de la pedagogía general y de la intervención pedagógica. (Touriñán y Sáez, 2015, p. 242)

De esta manera, la propuesta de ciudad educadora debe responder a un proyecto social y educativo que articule y entienda la ciudad con una doble condición: como ámbito de conocimiento y como ámbito de acción. Hablamos de la idea de acción como finalidad orientada a la consecución de las expectativas y los logros (como en el ejemplo que hemos apuntado del ApS). Para ello, la ciudad necesita ser percibida como un contexto cognoscible desde el que se crean conocimientos transculturales obtenidos de la realidad. Esto hace posible proponer experiencias que faciliten el contacto directo con la ciudad, para «después discutir y reflexionar sobre la experiencia, promocionar espacios de participación así como acciones vinculadas con el sentido de pertenencia e identidad» (Pérez, 2005, p. 133).

La concreción científico-social de la acción educativa en la ciudad propone unos paradigmas de acción circunscritos a tres parámetros: la actividad investigadora, la educativa y la formación del profesorado (Romero, 2004):

- El paradigma de acción investigadora, que se dedicará a concretar modelos efectivos para la acción educativa llevada a cabo en y desde la ciudad. ${ }^{15}$

15. A pesar de esto, no podemos ignorar lo que recuerda Houssage (1997, p. 93): «ningún investigador en educación debe erigir sus "verdades científicas" sobre la acción educativa como "matrices de verdad" para la acción». 
- El paradigma de acción del docente, encargado de implementar una acción educativa que pueda ser secuenciada en procesos de enseñanza y aprendizaje. ${ }^{16}$

- El paradigma de la formación del docente, basado en propuestas de investigación-acción capacitadas para explicar la complejidad ${ }^{17}$ de las acciones humanas y, por ende, las educativas.

La combinación de estos tres paradigmas y la colaboración de las instituciones políticas como administraciones que garantizan espacios de formación y participación ciudadana complementan el papel educativo de la ciudad, que no se reduce a lo inmediato, más bien sugiere comprensiones reflexivas que imbrican a la educación con su entorno, por eso va más allá de sus atribuciones habituales: «la ciudad se convierte en un continente de recursos educativos, [...] tiene una infraestructura pedagógica estable, formada por instituciones específicamente educativas (formales y no-formales)» (Pérez, 2005, p. 137), instituciones de las que podemos hacer uso y disfrute.

A la postre, la propuesta de ciudad educativa condiciona a una intencionalidad educativa que no debería radicar exclusivamente en $e l$ saber y el análisis de los agentes implicados, sino en una articulación pedagógica insertada en otros escenarios que puedan representar las diferentes estructuras básicas de la acción (humana): conceptualizar los nuevos espacios educativos, determinar las áreas de intervención y los elementos de apoyo que las diferentes cien-

16. En una investigación reciente centrada en detectar todo aquello que ofrecen sus localidades a docentes de dos ciudades tan distantes como Montevideo y Valencia, Huerta (2014, p. 145) concluye que «los docentes valoran positivamente la realidad de sus propias ciudades, defienden lo patrimonial y critican aquellos aspectos que consideran fallidos o mejorables. [...] Asumen su responsabilidad como formadores de las generaciones más jóvenes, y desean participar en la creación de una nueva conciencia urbana».

17. Von Foerster (1996) defiende que la dificultad de las ciencias humanas no se asienta en su menor medida de cientificidad en comparación a las ciencias naturales sino en la esencia de su finalidad y en lo inapropiado que resulta ser el método científico respecto a sus objetos de conocimiento. Incluso llega a afirmar que las ciencias duras tienen un éxito que puede considerarse como engañoso, puesto que el tipo de problemas que afrontan son simples (en contraposición a los de las ciencias humanas y sociales en general). No se puede profundizar ahora en este debate, ya que queda fuera de los objetivos del presente artículo. 
cias sociales aportan, definir los medios didácticos necesarios, aprovechar la multidimensionalidad de las fuentes de conocimiento digitales, etc.

La (pr)esencia antropológica de todo lo que contiene una ciudad vincula los objetivos de la educación hacia la orientación de la acción, es decir, hacia el mundo de vida del ser humano como ciudadano y como ser vivo que intuye, explora y ejecuta los significados que le va proporcionando la acción.

La instancia mediadora entre la pedagogía y la acción habrá que hallarla en prácticas convertidas en instancias creadoras de mundos de vida; lo relevante para que la ciudad educadora no quede reducida a buenas intenciones, entonces, será consolidar la estructuración y la presencia de los agentes y/o grupos sociales que conforman las redes sociales de las ciudades. Y también diseñar y promover campañas que ayuden a convencer a los equipos docentes de que:

La ciudad es un marco y un agente educador que, ante la tendencia a la concentración de poder, practica la opinión pública y la libertad; ante la tendencia al gregarismo, expresa pluralismo; ante la tendencia a distribuir desigualmente las posibilidades, defiende la ciudadanía; ante la tendencia al individualismo, se esfuerza por practicar la individualidad solidaria, permite formar personas sensibles tanto a sus deberes como a sus derechos. (Molas, 1990, p. 48)

De esta manera, entendemos la ciudad educadora como aquella capaz de establecer unas prácticas que desarrollan la educación en un contexto urbano dedicado a la integración, un contexto entendido como un eje de coordinación de saberes y actividades a través de las cuales el alumnado aprende, pero también tiene la posibilidad de forjar aportaciones conjuntas al bien común.

\section{Reflexiones finales}

La educación se encuentra en permanente transformación y necesita de múltiples saberes y espacios a los que debe complementar y convertir en fuentes de información productivas. Este artículo ha propuesto la ciudad educadora, una sugerencia pensada desde la relación escuela-ciudad que ambiciona el reconocimiento del saber hecho experiencia para ponerlo al servicio de un 
alumnado que pueda reconocer, analizar y transformar las redes de relaciones situadas y determinadas.

El objetivo de nuestra propuesta es que la ciudad no es únicamente un lugar para residir, sino que se puede aprender de ella, lo que implica una unidad de intenciones entre la escuela y la ciudad.

La ciudad educadora es un proyecto que, como ya se demostró en Turín en la década de los 70, reivindica lo colectivo e intenta confluir con la modernidad a partir de entender la educación como un hecho comunicativo cuyo desarrollo facilita la integración de todo su alumnado y la capacidad de influencia de la sociedad sobre sus propios horizontes, instaurando cambios de actitud y comportamientos en la ciudadanía y buscando, como fin último, la consolidación de la democracia como proyecto social colectivo. Sin embargo, conviene tener muy presente que:

Como todos los conceptos que, lejos de los perfectos e inamovibles del cielo platónico, se enraízan en la complejidad y fluencia de la realidad humana, el concepto de ciudad educadora cambia con el cambio mismo de la vida de las ciudades y sus habitantes; ello no implica un cambio que la «subordine» concesivamente a las crecientes presiones y dificultades de todo orden, pero sí un cambio que la «coordina» o adapta a las nuevas características y necesidades de las ciudades. Su genuino espíritu inicial no tiene por qué perderse en el cambio, porque desde su origen responde a invariantes humanas y sociales de fondo.

(Del Pozo, 2008, p. 25).

Más que perderse en los cambios, la ciudad educadora debe indagar en la vertiente educativa del espacio urbano y demandar una formación social basada en valores éticos que proporcionen al alumnado los valores de su acción y (una parte) del reconocimiento como persona social. Esto hará posible conjuntar los valores aprendidos en la escuela con los valores que se pueden ejercer en la ciudad, confluyendo, en sentido práctico, ambas realidades. Es entonces cuando cada práctica pedagógica concreta se convierte en un acto de enseñanza-aprendizaje en el que la ciudad conforma un proceso interdisciplinario donde el conocimiento del saber se adhiere a los pilares didácticos que guían la apropiación reflexiva de la urbe en sus diversos procesos: históricos, sociales, éticos, culturales, etc. (Rodríguez, 2008). 
No obstante, conviene tener muy presente que cada ciudad es distinta; cada ciudad va configurando unas proyecciones que el transcurso de la historia fosiliza en la conciencia de quienes la habitan y en un itinerario estructurado a partir de una encrucijada de caminos culturales y sociales muy complejos. Las ciudades actuales no siempre respiran como nos gustaría, puesto que problemas de diversa índole (presupuestarios, de convivencia, etc.), en muchas ocasiones, hacen que en la ciudad en la que vive nuestro alumnado tengamos la sensación de que no haya flujo de aire.

En consecuencia, la construcción como intersección pedagógica de la ciudad educadora debe marcarse como objetivo a tener muy presente que la finalidad de la acción educativa se bifurca entre su ejecución constitutiva como una parte más de la ciencia social y su capacidad de (re)construcción de la ciudad. A la primera la reemplaza la pedagogía; a la segunda, el ser humano. El reto es, cuando menos, apasionante.

\section{Referencias}

Ahedo, J. (2016). «La universidad: una escuela al servicio de la verdad». Revista Complutense de Educación, 2, pp. 517-532.

Ainscow, M. (2001). «Comprendiendo el desarrollo de escuelas inclusivas. Notas y referencias bibliográficas». Recuperado de: http://cursoestatalxxetapa.files.wordpress.com/2011/04/antologia-parte-3.pdf

Alfieri, F. (2005). «Turín, Ciudad Educadora». Educación y ciudad, 8, 2946.

Arellano, A. (2005). «Mirar la pedagogía desde tiempos inciertos». En: A. Arellano (Coord.) La educación en tiempos inciertos. Barcelona: Anthropos, pp. 79-92.

Beltrán, M. (2017). «Metodología para evaluar la vitalidad de un barrio. La enseñanza de estrategias locales». Kult-ur, 4, vol. 7, pp. 27-54.

Blackson, H. (2012). The Five Cs of Neighborhood Planning. Recuperado de: http://bettercities.net/news-opinion/blogs/howard-blackson/18813/fve-cs-neighborhood-planning

BorJa, J. y Castells, M. (1997). Lo local y lo global. La gestión de las ciudades en la era de la información. Madrid: Taurus.

- (2003). La ciudad conquistada. Madrid: Alianza. 
Bryon, P. y Gaona, G. (2005). «Ciudad Educadora: entre la realidad y la utopía». Educación y ciudad, 8, pp. 125-141.

Cabezudo, A. (2005). «Ciudad Educadora, un espacio para la democracia». Educación y ciudad, 8, pp. 77-88.

Calonge, F. (2017). «Estar cerca en la lejanía. El surgimiento de los entornos de vida en una periferia». Athenea Digital, 17(2), pp. 149-173.

Castro, J. O. (2005). «Ciudad educadora, he ahí el problema». Educación y ciudad, 8, 61-76.

Del Pozo, J. M. (2008). «El concepto de ciudad educadora, hoy». En: Educación y vida urbana. Madrid: Santillana, pp. 25-36.

Dewey, J. (1938). Experience and Education. Nueva York: Macmillan.

Dickerson, N. (2008). «Occupational and Residential Segregation. The Confluence of Two Systems of Inequality». Labor Studies Journal, 33(4), pp. 393-411.

Domangue, E., \& Carson, R. L. (2008). «Preparing Culturally Competent Teachers: Service-Learning and Physical Education Teacher Education». Journal of Teaching in Physical Education, 27(3), pp. 347367.

Figueras, P. (2008). «Ciudades Educadoras, una apuesta de futuro». En: Educación y vida urbana. Madrid: Santillana, pp. 19-24.

Garnique, F. y Gutiérrez, S. (2012). «Educación básica e inclusión: un estudio de representaciones sociales». Magis, 4(9), pp. 577-593.

Gimeno, J. (2013). En busca del sentido de la educación. Madrid: Morata.

Giraldo, F. (1995). «Paradigmas teóricos y modelos de desarrollo: La complejidad y la Política Urbana». En: Paradigmas teóricos y modelos de desarrollo en América Latina. Apuntes del Genes. Separata N. 2. Santa Fe de Bogotá, pp. 297-321.

Gozálvez, V. (2010). «Hacia una reconstrucción de la razón pedagógica». Teoría de la Educación. Revista Interuniversitaria, 22(2), pp. 1942.

Harvey, J. (2008). «Utopías dialécticas». En: Educación y vida urbana. Madrid: Santillana, pp. 45-52.

HeidegGer, M. (1989). Serenidad. Barcelona: Ediciones del Serbal.

Houssage, J. (1997). «Spécificité et dénégation de la pédagogie». Revue Française de Pédagogie, 120, pp. 83-97.

Huerta, R. (2014). «La mirada de los docentes hacia su ciudad : identidades urbanas y educación patrimonial». Pulso, 37, pp. 127-147. 
JACQuin, C. (2012). «Producir y habitar la periferia. Los nuevos conjuntos de vivienda de bajo costo en México (ZMVM)». Bulletin de l'Institut Français d'Études Andines, 41(3), pp. 389-415.

Kolb, D. A. (1984). Experiential Learning: Experience as the Source of Learning and Development. Englewood Cliffs, NJ:Prentice-Hall, Inc.

Limón, M. R. y Crespo, J. A. (2002). «Ciudad educadora y nuevos espacios de educación para la salud en las personas mayores». Revista Educación XXI,4, pp. 91-124.

Lindón, A. (1997). De la expansión urbana y la periferia metropolitana. Ciudad de Mexico: El Colegio Mexiquense.

Lozano, M.; Traver, J. y Sales, A. (2016). «La escuela en el barrio. Cartografiando las necesidades de cambio socioeducativo». Aularia, 2, pp. 13-20.

Malpas, Jeff (2004). Place and Experience. A Philosophical Topography. Cambridge: Cambridge University Press.

Molas, I. (1990). «La Ciudad y la Ciudadanía Democrática». En: La Ciudad Educadora. Barcelona: Ayuntamiento de Barcelona, pp. 41-45.

Molt, J. (2000). Histórias de vida, histórias de escola: elementos para una pedagogía da cidade. Vozes: Petrópolis.

Morin, E. (1998). Introducción al pensamiento complejo. Geodesia: Madrid.

Pallarès, M. y Planella, J. (2016). «Utopía, educación y cambio social transformador. De Hinkelammert a Habermas». Opción, Año 32, No. 79 , pp. 126-144.

- Chiva, O. (2017). La pedagogía de la presencia. Tecnologías digitales y aprendizaje-servicio. Barcelona: UOC.

— Traver, J. (2017). «Sobre las interpretaciones pedagógicas de Habermas y Rorty: más allá del modelo fundacionalista». Athenea Digital, 17(2), pp. 289-311.

Paupeiro, M. (2004). «Os cenários políticos e padagógicos de inovações político-pedagógicas na rede municipal de ensino de Porto Alegre». En J. Moll (Org.), Ciclos na escola, tempos na vida. Armed-Editora S/A Brasil, Porto Alegre.

Park, R. (2000). On Social Control and Collective Behaviour. Chicago: Chicago University Press.

Pérez, P. (2005). «¿La ciudad puede llegar a ser educadora?» Iconos. Revista de Ciencias Sociales, 23, pp. 127-140. 
Pinder, D. (2011). «Cities: Moving, Plugging in, Floating, Dissolving». En T. Cresswell \& P. Merriman (Eds.), Geographies of Mobilities. Practices, Spaces, Subjects, pp. 167-188. Farnham: Ashgate.

Rodríguez, J. (2000). «Formación ciudadana y urbana». En C. Miñana Blasco (Ed.), Interdisciplinariedad y Currículo, construcción de proyectos escuela-universidad. Universidad Nacional-Colombia: Bogotá.

- (2007). «Ciudad educadora. Una perspectiva política desde la complejidad». Revista Urbano, 16, pp. 29-49.

- (2008). «La participación como un acto educador y constructor de la Ciudad Educadora». Revista Iberoamericana de Educación, 42(2), pp. $1-22$.

— DíEz, E. J. (2017). «Ciudades educadoras: espacios de encuentro y convivencia antineoliberal». Intersticios: Revista Sociológica de Pensamiento Crítico, 11(1), pp. 69-81.

Romero, C. (2004). Conocimiento, acción y racionalidad en educación. Madrid: Biblioteca Nueva.

Strohmayer, U. (2011). «Bridges. Different Conditions of Mobile Possibilities». En T. Cresswell \& P. Merriman (Eds.), Geographies of Mobilities. Practices, Spaces, Subjects, pp. 119-136. Farnham: Ashgate.

Thrift, N. (2008). Non-Representational Theory. Space, Politics, Affect. London: Routledge.

Torres, J. A. (2009). «Análisis de los procesos de inclusión social y educativa: nuevos retos para suprimir las desigualdades y compartir las diferencias». Contextos Educativos, 12, pp. 31-56.

Touraine, A. (1988). ¿Qué empleo para los jóvenes? Madrid: Tecnos.

Touriñán, J. M y SÁez, R. (2015). La Mirada pedagógica. Teoría de la educación, metodología y focalizaciones. A Coruña: Andaviara.

Trilla, J. (1997). «Ciudades educadoras: bases conceptuales». En M. A. Sabbag, Ciudades Educadoras. Curitiba: UFPR-Brasil.

Von Foerster, H. (1996). Las semillas de la cibernética. Obras escogidas. Barcelona: Gedisa.

WALKer, A. (1997). «Introduction: The Strategy of Inequality». En A. Walker y C. Walker (Eds.), Britain Divided. The growth of social exclusion in the 1980s and 1990s. Londres: CPAG Ltd.

Walker, J. A. y Chaplin, S. (2002). Una introducción a la cultura visual. Barcelona: Octaedro. 
